\title{
Multi-focal metastatic papillary thyroid cancer to skeletal muscle with BRAFV600E mutation
}

\author{
Terry Shin ${ }^{1}$, Thanh D Hoang ${ }^{1}$, Sweeney P Shane ${ }^{2}$, Vinh QMai ${ }^{1}$ and Mohamed K.M. Shakir ${ }^{1}$ \\ ${ }^{1}$ Division of Endocrinology, Department of Medicine, Walter Reed National Military Medical Center, Bethesda, USA \\ ${ }^{2}$ Department of Pathology, Walter Reed National Military Medical Center, Bethesda, USA
}

\begin{abstract}
Methods: Report patient history and review of the literature.

\section{Introduction}

Skeletal muscle metastasis in papillary thyroid cancer is extremely rare with a total of 32 cases reported in the literature. We are reporting a patient with multi-focal skeletal muscle metastases from papillary thyroid cancer (PTC) with a documented BRAFV600E mutation.
\end{abstract}

Objective: Skeletal muscle metastasis in papillary thyroid cancer is extremely rare with a total of 32 cases reported in the literature. We are reporting a patient with multi-focal skeletal muscle metastases from papillary thyroid cancer (PTC) with a documented BRAFV600E mutation.

Results: A 52-year-old man underwent total thyroidectomy with classic PTC with a BRAFV600E mutation and confirmed skeletal metastasis.

Conclusion: The case describes a unique and rare presentation of metastatic PTC to skeletal muscle that requires a unique approach to diagnosis and management.

\section{Case report}

A 52-year-old man underwent total thyroidectomy for an incidental thyroid nodule that was confirmed PTC via fine needle biopsy. Pathology confirmed a $5.5 \mathrm{~cm}$ papillary thyroid cancer (PTC) without aggressive histology or local extension. Five out of seven cervical lymph nodes were positive at level 6 of cervical lymph nodes. After surgery, he was treated with $177 \mathrm{mCi}$ of $\mathrm{I}^{131}$. Post-treatment scan revealed right lower neck uptake and multi-focal thyroid bed uptake.

Two years later, he demonstrated detectable basal thyroglobulin (Tg) elevation with negative $\mathrm{Tg}$ antibody. Thyrogen stimulated $\mathrm{Tg}$ rose to $60 \mathrm{ng} / \mathrm{mL}$ (ref. 2.0-35). However, iodine $\left(\mathrm{I}^{131}\right)$ whole body scan revealed no evidence of uptake in thyroid bed or other sites. ${ }^{18} \mathrm{~F}$-fluorodeoxy positon emission computerized tomography scan $\left({ }^{18} \mathrm{~F}\right.$ DG PET-CT) demonstrated evidence of a $1.3 \mathrm{~cm}$ residual neck mass suggestive of residual thyroid cancer. Patient underwent repeat 156 $\mathrm{mCi}$ of $\mathrm{I}^{131}$ ablation and post-treatment scan reported focus uptake in the right neck.

The following year, he was found to have persistent disease (1.5 $\mathrm{cm}$ nodule in thyroid bed) confirmed via magnetic resonance imaging (MRI) of the neck. Repeat Thyrogen ${ }^{\mathrm{TM}}$ stimulated serum Tg was $14 \mathrm{ng} /$ $\mathrm{mL}$. He subsequently received his third dose of $293 \mathrm{mCi} \mathrm{I}^{131}$ treatment with a total of $626 \mathrm{mCi}$.

The next year after his third radioactive iodine treatment surveillance ${ }^{18} \mathrm{~F}$-FDG PET-CT scan reported a neck mass confirmed by neck MRI $(1.5-2.0 \mathrm{~cm})$ now involving the esophagus with rising serum $\mathrm{Tg}$ levels. He underwent thyroid mass resection followed by external beam radiation therapy. Pathology of the neck mass confirmed metastatic PTC.

Two years later, $\mathrm{I}^{131}$ WBS reported no evidence of disease but FDG PET-CT reported a $3.7 \times 2.7 \times 2.6 \mathrm{~cm}$ hypermetabolic mass in the right upper arm as well as multiple intramuscular hypermetabolic lesions in the sternum, left trapezius, left gluteus maximus, left hip abductors, and persistent activity within the thyroid bed, $2.3 \mathrm{~cm}$ right hypermetabolic lung nodules was also identified and suspicious for metastatic disease (Figure 1). He underwent partial sternal resection with removal of the mass from the upper right arm. Tissue examination revealed randomly oriented papillae lined with cuboidal cells with overlapping and crowded nuclei (Figure 2A-B) consistent with metastatic PTC to muscle. BRAFV600E mutation was identified in the right arm metastatic tissue sample.

Due to the lack of response with RAI, the patient was offered a trial of BRAFV600E inhibitors. He declined treatment because of work obligations overseas and since he was overall asymptomatic despite his tumor burden. He opted for surveillance with consideration for future trials if his disease continued to demonstrate progression.

\section{Discussion}

There is a total of 32 reported cases of skeletal metastases from PTC in the literature over the past 110 years [1]. Generally, muscle metastases from solid tumors are rare. The majority of skeletal muscle metastases are from lung (25.1\%), gastrointestinal (21\%), and urologic tumors (13.2\%) [2]. Muscle metastases from thyroid cancer is scarcer

*Correspondence to: Thanh D Hoang, Division of Endocrinology, Walter Reed National Military Medical Center, 8901 Wisconsin Ave, Bethesda, MD 20889, USA, E-mail: thanh.d.hoang.mil@mail.mil

Received: February 02, 2020; Accepted: March 18, 2020; Published: March 23, 2020 


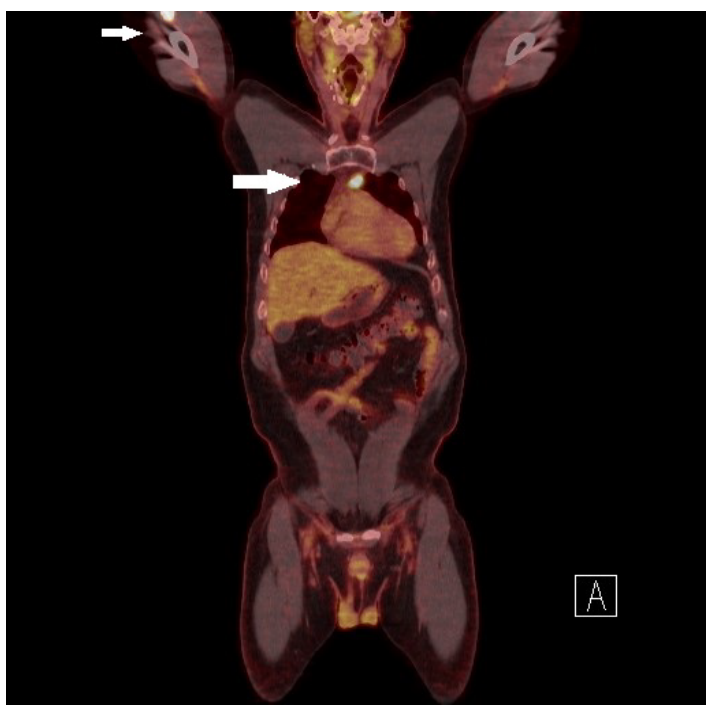

Figure 1. Arrows highlight metastatic PTC identified on PET-CT and PET

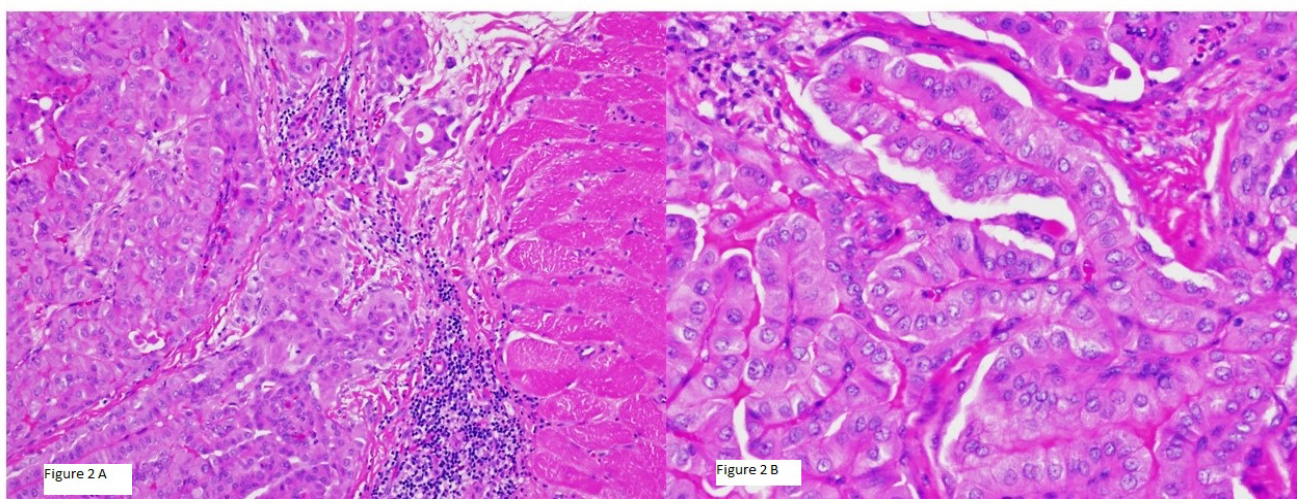

Figure 2. Pathology of right arm mass resection: left image with PTC invading muscle tissue with right image showing high powered view of classic PTC

and reported at approximately $3.7 \%$ [2]. The calculated probability of detecting muscle metastases in PTC is 4/1,000,000,000 [1].

There are several theories and studies that may explain the rarity of muscle metastases. Skeletal muscle produces several anti-tumor factors that are known to inhibit proliferation of malignant cells and these include low molecular weight factor, adenosine, leukemia inhibitor factor, and interleukin [3-6]. It is postulated that the microvasculature of skeletal muscle may biomechanically be damaged via tumor cells [5]. Variable blood flow within skeletal muscle may also limit tumor growth [6,7]. Lastly, the muscle's ability to remove lactic acid inhibit may also inhibit tumor neovascularization $[2,6]$.

The pathophysiology behind the spread of metastatic tumor into muscle is believed to occur through several mechanisms. One is through direct hematogenous spread of cancer cells, and studies have demonstrated tumor cells directly spreading via the arterial circulation [7]. Another process is through lymphatic spread to intramuscular lymph nodes $[2,8]$. There is also the possibility of skeletal muscle metastases occurring through perineural spread [9]. It may be hypothesized that given the propensity for PTC metastases to spread through regional lymph nodes, spread of PTC to muscle may occur through lymphatic proliferation.

The most common site for distal metastatic spread in PTC is the lungs. The second common is bone and then the brain [10]. There are other rare instances where PTC was reportedly found in the adrenals, kidney, ovary, orbit, and sphenoid sinus [10]. Only a third of the patients had pain from the muscle metastases from thyroid cancer, and the most frequent site of involvement was the gluteus muscle [1]. Median survival was found to be reduced by nearly half in patients with skeletal muscle metastases compared to other organ thyroid metastases [1]

Our patient was identified to have the BRAFV600E mutation. BRAF (kinase-activating mutation in the V-raf murine sarcoma viral oncogene homolog B1) is gene that encodes for a protein in the RAS/ MAPK signaling cascade. The RAS/MAPK pathway regulates cellular proliferation, differentiation, migration, and apoptosis [11]. Alteration in BRAF affects the pathway and leads to tumor cell proliferation or loss of differentiation. ${ }^{12}$ This proto-oncogene mutation was first discovered in 2002 as potentiator of various cancers [12]. The majority of BRAF mutations (90\%) are due to a single amino substitution at codon 600 designated as BRAFV600E [13]. This BRAFV600E mutation is found in $43.8 \%$ papillary thyroid cancers and present in approximately $55-75 \%$ classical type PTC [14-16].

Papillary thyroid cancers that harbor BRAFV600E mutations are found to be resistant to radioactive iodine due to decreased expression of the sodium-iodide symporter $[17,18]$. Case reports on papillary thyroid cancer with muscle metastasis reported poor response to radioactive iodine [10]. The patient reported here demonstrated a poor 
response to treatment with $I^{131}$. Radioiodine scan with postoperative serum Tg levels are the standard for detecting metastatic disease [19]. However, the $\mathrm{I}^{131}$ WBS is negative in $20 \%$ of patient with disseminated thyroid cancer and several case reports have demonstrated negative radioiodine scans in these patients [20]. ${ }^{18} \mathrm{~F}$ FDG-PET scan has been shown to be a valuable tool in identifying and localizing recurrences when serum Tg is elevated with a negative $\mathrm{I}^{131}$ WBS.

Multitargeted kinase inhibitors are now FDA approved for advanced or metastatic-radioactive iodine refractory thyroid cancer with extended progression free survival (PFS) ranging from 5 to 14.7 months $[21,22]$. However, the toxicity associated with tyrosine kinase inhibitors may limit its use in only advanced cases and may not be suitable for patients who are asymptomatic or with indolent slow growing metastases. Identification of BRAF mutation are may serve as an important marker in the treatment of unresectable or metastaticradioactive iodine resistant papillary thyroid cancer to skeletal muscle and these patients may be treated with of BRAF inhibitors. Though treatment with MEK (mitogen-activated protein kinase enzymes MEK1 and/or MEK2) inhibitors such as selumentinib have been tried in PTC, recent trials specifically targeting papillary thyroid cancer with the BRAFV600E mutation are ongoing [23]. The use of selective BRAF inhibitors may be a potential option in our patient although he is asymptomatic but demonstrates ongoing progression of his disease. Two selective BRAF inhibitors, dabrafenib and vemurafenib have demonstrated some success by restoring sensitivity to radioactive iodine by redifferentiation of iodine -refractory thyroid cancer $[24,25]$. The patient is planned for the possibility of treatment with BRAFV600E in the future.

\section{Conclusion}

In summary, we describe a rare case of metastatic PTC to skeletal muscle that was found to harbor the BRAFV600E mutation. Clinicians should consider genetic testing in patients with radioiodine resistant thyroid cancer given the potential change in surveillance and treatment strategies in this unique patient population.

\section{Acknowledgment}

The views expressed in this article are those of the authors and do not reflect the official policy or position of the Department of the Army, Department of the Navy, Department of Defense, or the U.S. Government.

\section{Disclosure}

The authors have no multiplicity of interest to disclose.

\section{References}

1. Herbowski L (2018) Skeletal muscle metastases from papillary and follicular thyroid carcinomas: An extensive review of the literature. Oncol Lett 15: 7083-7089. [Crossref]

2. Surov A, Kohler J, Wienke A, Gufler H, Bach AG, et al. (2014) Muscle metastases: comparison of features in different primary tumours. Cancer Imaging 14: 21. [Crossref]

3. Djaldetti M, Sredni B, Zigelman R, Verber M, Fishman P (1996) Muscle cells produce a low molecular weight factor with anti-cancer activity. Clin Exp Metastasis 14: 189196. [Crossref]

4. Kurek JB, Nouri S, Kannourakis G, Murphy M, Austin L (1996) Leukemia inhibitory factor and interleukin- 6 are produced by diseased and regenerating skeletal muscle. Muscle Nerve 19: 1291-1301. [Crossref]

5. Weiss L, Schmid-Schonbein GW (1989) Biomechanical interactions of cancer cells with the microvasculature during metastasis. Cell Biophys 14: 187-215.

6. Seely S (1980) Possible reasons for the high resistance of muscle to cancer. Med Hypotheses 6: 133-137. [Crossref]
7. Pearson CM (1959) Incidence and type of pathologic alterations observed in muscle in a routine autopsy survey. Neurology 9: 757-766.

8. Lee JK, Glazer HS (1986) Psoas muscle disorders: MR imaging. Radiology 160: 683687. [Crossref]

9. Blandino A, Gaeta M, Minutoli F, Pandolfo I (2000) CT and MR findings in neoplastic perineural spread along the vidian nerve. Eur Radiol 10: 521-526. [Crossref]

10. Bae SY, Lee SK, Koo MY, Hur SM, Choi MY, et al. (2011) Distant, solitary skeletal muscle metastasis in recurrent papillary thyroid carcinoma. Thyroid 21: 1027-1031. [Crossref]

11. Aoki Y, Niihori T, Narumi Y, Kure S, Matsubara Y (2008) The RAS/MAPK syndromes novel roles of the RAS pathway in human genetic disorders. Hum Mutat 29: 992-1006. [Crossref]

12. Davies H, Bignell GR, Cox C, Stephens P, Edkins S, et al. (2002) Mutations of the BRAF gene in human cancer. Nature 417: 949-954. [Crossref]

13. Ritterhouse LL, Barletta JA (2015) BRAF V600E mutation-specific antibody: A review. Semin Diagn Pathol 32: 400-408. [Crossref]

14. Adeniran AJ, Zhu Z, Gandhi M, Steward DL, Fidler JP, et al. (2006) Correlation between genetic alterations and microscopic features, clinical manifestations, and prognostic characteristics of thyroid papillary carcinomas. Am J Surg Pathol 30: 216222. [Crossref]

15. Jung CK, Little MP, Lubin JH, Brenner AV, Wells SA Jr, et al. (2014) The increase in thyroid cancer incidence during the last four decades is accompanied by a high frequency of BRAF mutations and a sharp increase in RAS mutations. $J$ Clin Endocrinol Metab 99: E276-285. [Crossref]

16. Lupi C, Giannini R, Ugolini C, Proietti A, Berti P, et al. (2007) Association of BRAF V600E mutation with poor clinicopathological outcomes in 500 consecutive cases of papillary thyroid carcinoma. J Clin Endocrinol Metab 92: 4085-4090. [Crossref]

17. Romei C, Ciampi R, Faviana P, Agate L, Molinaro E, et al. (2008) BRAFV600E mutation, but not RET/PTC rearrangements, is correlated with a lower expression of both thyroperoxidase and sodium iodide symporter genes in papillary thyroid cancer. Endocr Relat Cancer 15: 511-520. [Crossref]

18. Riesco-Eizaguirre G, Gutierrez-Martinez P, Garcia-Cabezas MA, Nistal M, Santisteban $\mathrm{P}$ (2006) The oncogene BRAF V600E is associated with a high risk of recurrence and less differentiated papillary thyroid carcinoma due to the impairment of $\mathrm{Na}+/ \mathrm{I}-$ targeting to the membrane. Endocr Relat Cancer 13: 257-269. [Crossref]

19. Haugen BR, Alexander EK, Bible KC, et al. (2016) 2015 American thyroid association management guidelines for adult patients with thyroid nodules and differentiated thyroid cancer: The American thyroid association guidelines task force on thyroid nodules and differentiated thyroid cancer. Thyroid 26: 1-133. [Crossref]

20. Frilling A, Tecklenborg K, Gorges R, Weber F, Clausen M, et al. (2001) Preoperative diagnostic value of $[(18) \mathrm{F}]$ fluorodeoxyglucose positron emission tomography in patients with radioiodine-negative recurrent well-differentiated thyroid carcinoma. Ann Surg 234: 804-811. [Crossref]

21. Brose MS, Nutting CM, Jarzab B, Elisei R, Siena S, et al. (2014) Sorafenib in radioactive iodine-refractory, locally advanced or metastatic differentiated thyroid cancer: A randomised, double-blind, phase 3 trial. Lancet 384: 319-328. [Crossref]

22. Schlumberger M, Tahara M, Wirth LJ, Robinson B, Brose MS, et al. (2015) Lenvatinib versus placebo in radioiodine-refractory thyroid cancer. $N$ Engl J Med 372: 621-630.

23. Chakravarty D, Santos E, Ryder M, Knauf JA, Liao XH, et al. (2011) Small-molecule MAPK inhibitors restore radioiodine incorporation in mouse thyroid cancers with conditional BRAF activation. J Clin Invest 121: 4700-4711. [Crossref]

24. Dadu R, Shah K, Busaidy NL (2015) Efficacy and tolerability of vemurafenib in patients with BRAF(V600E) -positive papillary thyroid cancer: M.D. Anderson Cancer Center off label experience. J Clin Endocrinol Metab 100: E77-81.

25. Rothenberg SM, Daniels GH, Wirth LJ (2015) Redifferentiation of iodine-refractory BRAF V600E-mutant metastatic papillary thyroid cancer with dabrafenib-response. Clin Cancer Res 21: 5640-5641

Copyright: (C2020 Shin T. This is an open-access article distributed under the terms of the Creative Commons Attribution License, which permits unrestricted use, distribution, and reproduction in any medium, provided the original author and source are credited. 